\title{
Soft Nanolithography by Polymer Fibers
}

\author{
Deyu Tu, Stefano Pagliara, Andrea Camposeo, Giovanni Potente, Elisa Mele, \\ Roberto Cingolani, and Dario Pisignano*
}

We report on the use of polymer fibers for large-area soft nanolithography on organic and inorganic surfaces with $50 \mathrm{~nm}$ resolution. The morphology of fibers and of the corresponding patterned gap is investigated, demonstrating a lateral dimension downscaling of up to nine times, which greatly increases the achieved resolution during pattern transfer. In this way, we realize polymer field effect transistors with channel length and width as low as $\mathbf{2 5 0} \mathrm{nm}$ that are expected to show transistor transition frequency up to a few $\mathrm{MHz}$, and are thus exploitable as low-cost radio-frequency identification devices. to pattern elastomers as perfluoropolyether (PFPE)-urethane dimethacrylate, ${ }^{[9]}$ exhibiting high resistance to organic solvents, and nanoscale gap Au electrodes as low as $50 \mathrm{~nm}$. In this way, we realize fieldeffect transistors (FETs), by (i) spin-casting the conjugated polymer, poly(3-hexylthiophene) (P3HT) or (ii) depositing P3HT fibers on nanogap electrodes, using particle replication in nonwetting templates (PRINT). ${ }^{[2,9]}$

\section{Introduction}

Soft lithographies provide low-cost and high-throughput patterning approaches to the ongoing miniaturization of devices in electronics, ${ }^{[1]}$ photonics, ${ }^{[2]}$ biotechnology, ${ }^{[3,4]}$ and microfluidics. ${ }^{[5]}$ These technologies rely on masters, realized by conventional lithography, ${ }^{[6,7]}$ or on nanostructures replicated into elastomeric elements. ${ }^{[8-10]}$ For instance, carbon nanotubes (CNTs) are used to pattern elastomers ${ }^{[1]}$ and metals ${ }^{[12]}$ with molecular-scale resolution. However, low-cost and large-area nanopatterning still remains an open issue, mainly due to the CNT limited length $\left(10-10^{2} \mu \mathrm{m}\right){ }^{[11]}$ Soft nano-lithography (SNL) by polymer fibers realized through electrospinning $(\mathrm{ES})^{[13]}$ offers a simple route to large area patterning. ${ }^{[14]}$ Here, we report on electrospun fibers

Dr. D. Tu, S. Pagliara, Dr. A. Camposeo, G. Potente, Dr. D. Pisignano National Nanotechnology Laboratory of Consiglio Nazionale delle Ricerche-Istituto Nanoscienze

Università del Salento

via Arnesano, I-73100 Lecce, Italy

E-mail: dario.pisignano@unisalento.it

Prof. R. R. Cingolani

Istituto Italiano di Tecnologia (I.I.T.)

via Morego 30, I-16163, Genova, Italy

Dr. E. E. Mele

Istituto Italiano di Tecnologia (I.I.T.)

Center for Biomolecular Nanotechnologies

via Barsanti 1, I-73010, Arnesano, Lecce, Italy

Dr. D. Pisignano

Dipartimento di Ingegneria dell'Innovazione

Università del Salento

via Arnesano, I-73100 Lecce, Italy

G. Potente

Istituto Superiore di Formazione Interdisciplinare ISUFI

Università del Salento

via Arnesano, I-73100 Lecce, Italy

DOI: 10.1002/adfm.201001901

\section{Results and Discussion}

Arrays of uniaxially aligned fibers are electrospun from a formic acid solution of poly(methylmethacrylate) (PMMA) across parallel Al-coated glass slides with a $1.5 \mathrm{~cm}$-wide gap (Figure 1a), and then transferred onto the top $\mathrm{SiO}_{2}$ surface of a $1 \mathrm{~cm} \times 1 \mathrm{~cm} \mathrm{n}$-type $\mathrm{Si}$ substrate (Figure $1 \mathrm{~b}$ ). Then, a layer of $\mathrm{Cr} / \mathrm{Au}(5 \mathrm{~nm} / 45 \mathrm{~nm}$ ) is deposited over the fibers by physical vapor deposition (PVD) through shadow masks with a grid of about 250 square holes, each of $100 \mu \mathrm{m}$ side length, separated by $25 \mu \mathrm{m}$ wide bars (Figure 1c). Polymer fibers are lifted off by sonication in isopropanol, or removed by oxygen plasma, breaking the continuity in the metal and exposing $\mathrm{SiO}_{2}$ (Figure 1d), thus obtaining an array of nanogap electrodes. The used fibers are long (around $1 \mathrm{~cm}$ ), therefore, the production yield of the process in terms of fabricated electrodes is only limited by the linear density of the holes in the initial shadow mask $\left[8 \mathrm{~mm}^{-1}\right.$, thus providing up to 8 couples of electrodes per $\mathrm{mm}$, per each electrospun fiber, with a yield (couples of electrodes/initial holes in the line) as high as $90 \%$, depending on the degree of mutual alignment between the deposited fiber and the array of holes in the grid], and by the number of aligned fibers deposited simultaneously. Typically, in a single SNL step, more than 20 couples of electrodes are created in parallel by a single ES fiber, and can be used for realizing organic electronic devices (Figure 1e). Improving the set-up ${ }^{[15,16]}$ to carefully control the alignment degree and the density of the deposited fibers, and using masks with a higher density of holes could allow one to realize from many tens to a few hundreds of nanoscale gap electrodes in single runs.

Alternatively, the fibers array can be first replicated in elastomers (Figure 1b). Poly(dimethylsiloxane) (PDMS) or PFPEurethane dimethacrylate is cast on fibers preliminarily treated by hexamethyldisilizane (HMDS) vapor, ${ }^{[17]}$ polymerized in situ (Figure 1f) and patterned by parallel nanochannels (Figure 1g). 


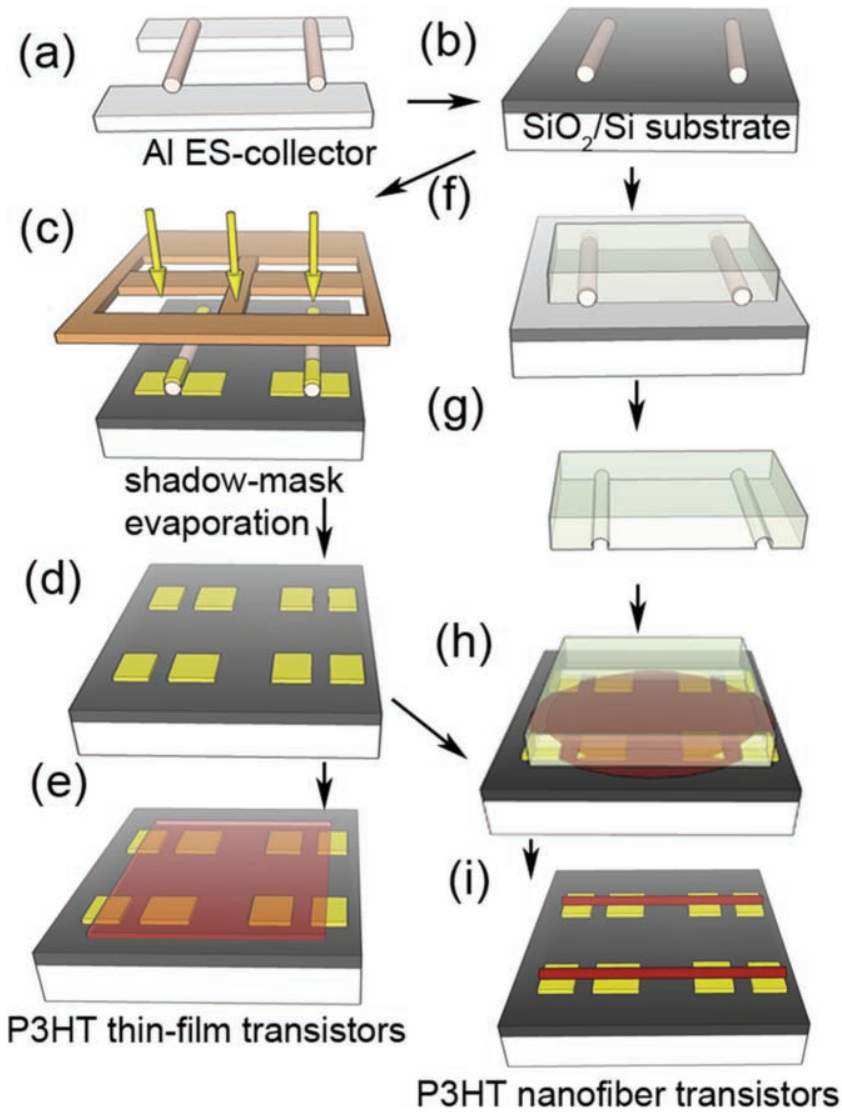

Figure 1. Soft nano-lithography (SNL) by polymer fibers. a) Deposition of uniaxially aligned arrays of fibers across parallel slides with Al coating, by electrospinning (ES). b) Fiber array mechanical transfer to the $\mathrm{SiO}_{2} / \mathrm{Si}$ substrate. $\mathrm{c}-\mathrm{e}$ ) SNL on inorganics: c) metal evaporation by PVD through a copper-grid shadow mask, d) fibers lift-off allowing to obtain arrays of electrodes, and e) device realization by spin-casting $\mathrm{P} 3 \mathrm{HT}$ as active material on the electrodes. $\mathrm{f}-\mathrm{i}) \mathrm{SNL}$ on organics: $\mathrm{f}$ ) on-fiber casting and in situ elastomer polymerization, g) elastomer peeling-off, $h$ ) pattern transfer to a conjugated polymer via solvent-resistant PRINT, and i) resulting nanofiber lying on electrodes.

Another option is using oxygen plasma to gently remove the fiber material (not treated by HMDS) trapped in the elastomer without damaging the replicated pattern. Finally, a droplet of P3HT toluene solution is placed between the PFPE-urethane dimethacrylate replica and the electrodes (Figure 1h). The organic solution, which does not wet PFPE-urethane dimethacrylate, remains confined within the recessed features of the mold, thus forming P3HT nanofibers upon toluene evaporation (Figure 1i). In fact, PFPE derivatives have many advantages over other elastomers such as PDMS, ${ }^{[2,9,10]}$ such as enabling high resolution and aspect ratio without structural collapses, and no swelling in non-polar solvents. This is a drawback of PDMS which prevents its application to many conjugated polymers. In addition, in contrast to other processes as soft molding, ${ }^{[18]}$ PRINT by PFPE-urethane-methacrylates is entirely performed at room temperature, thus being especially suitable for retaining the conduction properties of organic semiconductors.

In SNL on PDMS, we find that thin polymer fibers (diameter $<500 \mathrm{~nm}$ ) without HDMS treatment generally remain trapped
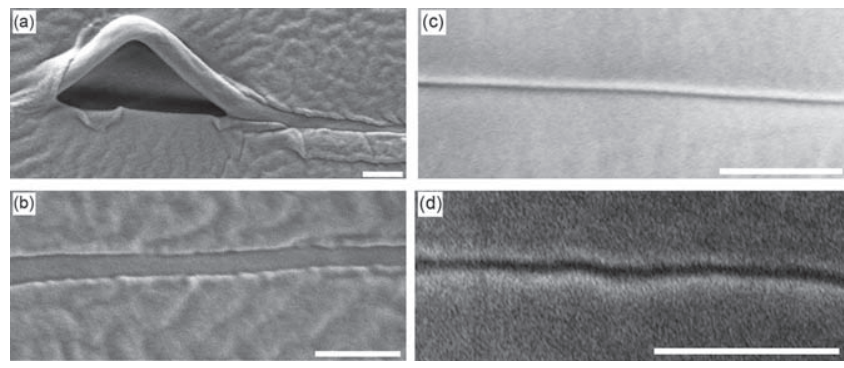

Figure 2. SEM micrographs of replicated features into PDMS. Bars = $1 \mu \mathrm{m}$. a) Failed mechanical peeling-off of a fiber (not previously treated by HDMS). Features width $=200 \mathrm{~nm}$ (b) and $50 \mathrm{~nm}$ (c and d).

into the elastomeric element and only partially detach from the replica surface (Figure 2a). HMDS-treated fibers are, in contrast, easily peeled-off, defining nanochannels in the replicas (Figure $2 \mathrm{~b}$ and $2 \mathrm{c}$ ), with a minimum lateral size of as low as $50 \mathrm{~nm}$ (Figure 2d).

Figure $3 \mathrm{a}$ and $3 \mathrm{~b}$ show electrodes with a width (W) of $100 \mu \mathrm{m}$ and gap length $(L)$ of $500 \mathrm{~nm}$ and $150 \mathrm{~nm}$, respectively. Y-shaped, crossed or straight features can be realized. In particular, Y-shaped planar features are realized by the accidental splitting of a single fiber into two thinner wires through ES fields $>1 \mathrm{kV} \mathrm{cm}^{-1}$ (Figure 3c). We find that the number of such splitted fibers increases with the ES field intensity. ${ }^{[15]}$ A geometry with four co-planar electrodes is derived from a single $\mathrm{Au}$ region (Figure $3 \mathrm{~d}$ ), by sequentially collecting fibers aligned along orthogonal directions. The ES facilitates the careful control of the diameter from tens of $\mathrm{nm}$ to the micrometer scale. For instance, with a solution concentration of a few $\mathrm{mM}$, a needlecollector distance of a few $\mathrm{cm}$ and low fields $\left(<0.5 \mathrm{kV} \mathrm{cm}^{-1}\right)$, fibers with a diameter of up to a few $\mu \mathrm{m}$ can be obtained. For our application, a crucial issue is the uniformity of the diameter along the fiber axis. To this aim, we use formic acid as a solvent of high dielectric constant, disfavoring the formation of beads. ${ }^{[19]}$ Typical fiber arrays on areas up to many $\mathrm{mm}^{2}$
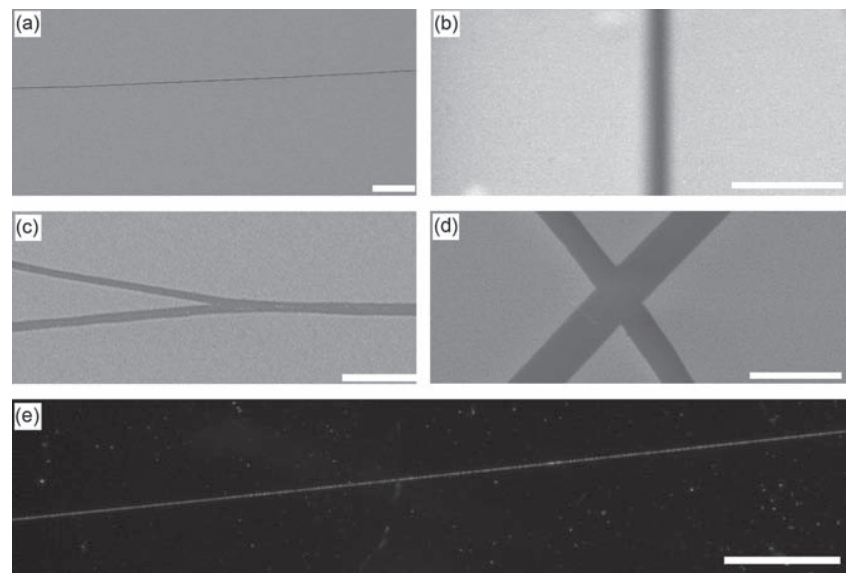

Figure 3. a-d) SEM micrographs of Au electrodes produced by SNL. a) A gap of $500 \mathrm{~nm}$ (scale bar $=20 \mu \mathrm{m})$. b) A gap of $150 \mathrm{~nm}($ bar $=2 \mu \mathrm{m})$. c) A "Y-shaped" gap (bar $=10 \mu \mathrm{m})$. d) Crossed planar gaps $($ bar $=2 \mu \mathrm{m})$. e) Stereomicroscope image of a $1 \mathrm{~cm}$-long, $600 \mathrm{~nm}$-wide gap (bright) realized by fiber-based SNL, between Au electrodes (dark). Bar $=1 \mathrm{~mm}$. 

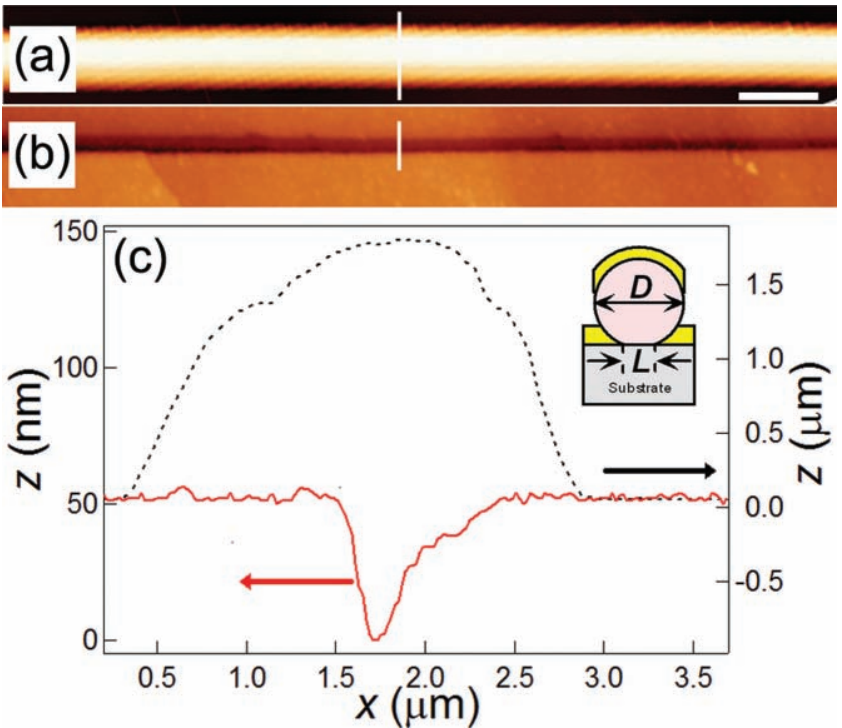

Figure 4. AFM planar views of a polymer fiber. a) After metal deposition. b) The resulting $180 \mathrm{~nm}$ gap electrodes after fiber removal (marker bar $=2 \mu \mathrm{m})$. c) Corresponding cross-sections profiles along the white lines in (a) and (b), represented as dashed (right vertical scale) and solid (left scale) lines, respectively. Inset: Scheme of metal spreading underneath cylindrical polymer fiber. $D$ : fiber diameter. $L$ : resulting electrodes separation.

exhibit diameter distributions of around 15\% of the average value (which is reflected in variations below $0.1 \%$ in terms of channel length/width ratio for our typical device geometry, see below). Furthermore, the highly flexible fibers can be deposited and replicated on surfaces in aligned, straight positions over many mm (Figure 3e). This approach can be pursued further to produce helical ${ }^{[20]}$ or aligned assemblies, ${ }^{[16]}$ and light-emitting nanofibers. ${ }^{[21-23]}$

Atomic force microscopy (AFM) is carried out on metalcoated fibers and on the corresponding nanoscale gaps on $\mathrm{SiO}_{2}$. The planar view (Figure 4a) and height profile (Figure 4c) show an average fiber diameter of 1.6-1.8 $\mu \mathrm{m}$. Upon fiber removal (Figure 4b), a U-shaped profile is obtained on Au (solid line in Figure $4 \mathrm{c})$, with an electrode height $(h)$ of $(45 \pm 5) \mathrm{nm}$, close to the nominal metal thickness. The average gap was $180 \pm 10 \mathrm{~nm}$, calculated by averaging the width values at half the maximum height of the AFM profiles measured at different points over a length of $100 \mu \mathrm{m}$. A relevant effect is associated with the lateral spreading of the metal vapor underneath the fiber during PVD (inset in Figure 4c). Given the low metal thickness $(<50 \mathrm{~nm})$, only the bottom circular sector of the fiber is effectively involved in the pattern transfer, which explains the observed reduction of the lateral dimension (up to 9 times) from the fiber to the metal gap. Using AFM, we also investigate the homogeneity of the obtained gaps, whose width changes by about $20 \%$ of its mean value along a $1 \mathrm{~mm}$ section (Figure 5), in agreement with the original variations observed in the fiber diameters.

Importantly, the gaps are electrically disconnected. To investigate this issue in depth, we produce electrodes separated by $70 \mathrm{~nm}$, whose profile is inspected by AFM over about $100 \mu \mathrm{m}$, together with the polymer nanofiber used as shadow mask

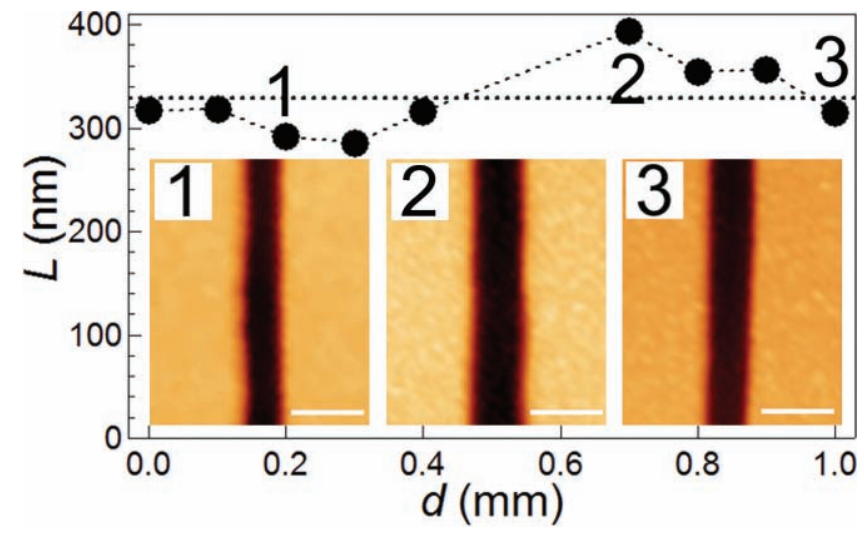

Figure 5. Electrodes separation $(330 \pm 35 \mathrm{~nm})$, measured along the gap length, and AFM micrographs corresponding to points 1, 2 and 3 , respectively (insets, bar $=500 \mathrm{~nm}$ ).

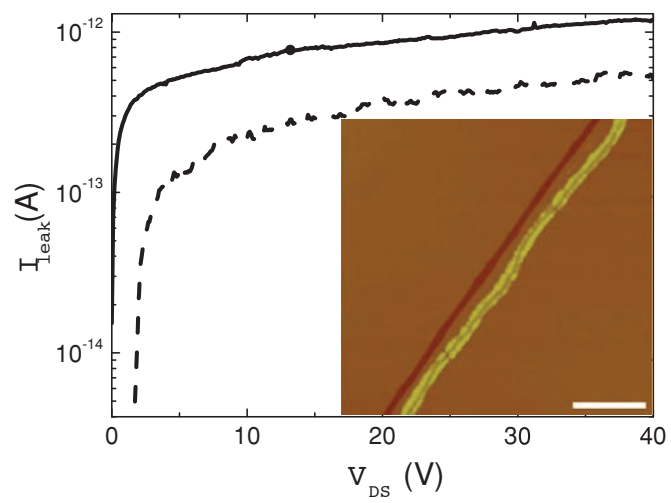

Figure 6. Current through electrodes separated by an SNL-made gap (solid curve) of $70 \mathrm{~nm}$, and by a shadow mask-made gap of $7 \mu \mathrm{m}$ (dashed curve). Inset: AFM image of the nanogap and of its templating polymer fiber. Marker $=2 \mu \mathrm{m}$.

during PVD (inset of Figure 6). The average fiber diameter is around $220 \mathrm{~nm}$. Electrical measurements, carried out by biasing the two resulting electrodes by up to $40 \mathrm{~V}$, provide evidence of the good insulating properties of the obtained gap. The leakage current $(\leq 1 \mathrm{pA})$ is slightly higher than that found for electrodes separated by $7 \mu \mathrm{m}$ realized through conventional shadow masks (Figure 6), and is well-suited for realizing organic FETs.

Polymer FETsare fabricated on SNL-made gaps $(L=400 \mathrm{~nm}$, $W=100 \mu \mathrm{m})$ by spin-coating $50 \mathrm{~nm}$-thick P3HT films. Similar devices with microgaps $(L=25 \mu \mathrm{m}, W=100 \mu \mathrm{m})$ exhibit a significant saturation above a drain-source voltage $\left(V_{\mathrm{DS}}\right)$ of $-30 \mathrm{~V}$ for gate-source voltage $\left(V_{\mathrm{GS}}\right)$ decreasing from +10 to $-10 \mathrm{~V}$ (Figure 7a), whereas the current-voltage characteristics $\left[I_{\mathrm{DS}}\left(V_{\mathrm{DS}}\right)\right]$ of nanogap devices do not exhibit such a saturation (Figure 7b). This is typical of short-channel effects related to downscaling. ${ }^{24]}$ The corresponding transfer characteristics, $I_{\mathrm{DS}}\left(V_{\mathrm{GS}}\right)$, at $V_{\mathrm{DS}}=-40 \mathrm{~V}$, together with the curves, $\left|I_{\mathrm{DS}}\right|^{1 / 2}\left(V_{\mathrm{GS}}\right)$, of micro- and nanogap devices are displayed in Figure $7 \mathrm{c}$ and $7 d$. SNL-made FETs show a $I_{\text {on }} / I_{\text {off }}$ switching ratio, given by the maximum $\left(I_{\mathrm{on}}\right)$ and the minimum $\left(I_{\text {off }}\right)$ values of $I_{\mathrm{DS}}$ in the 

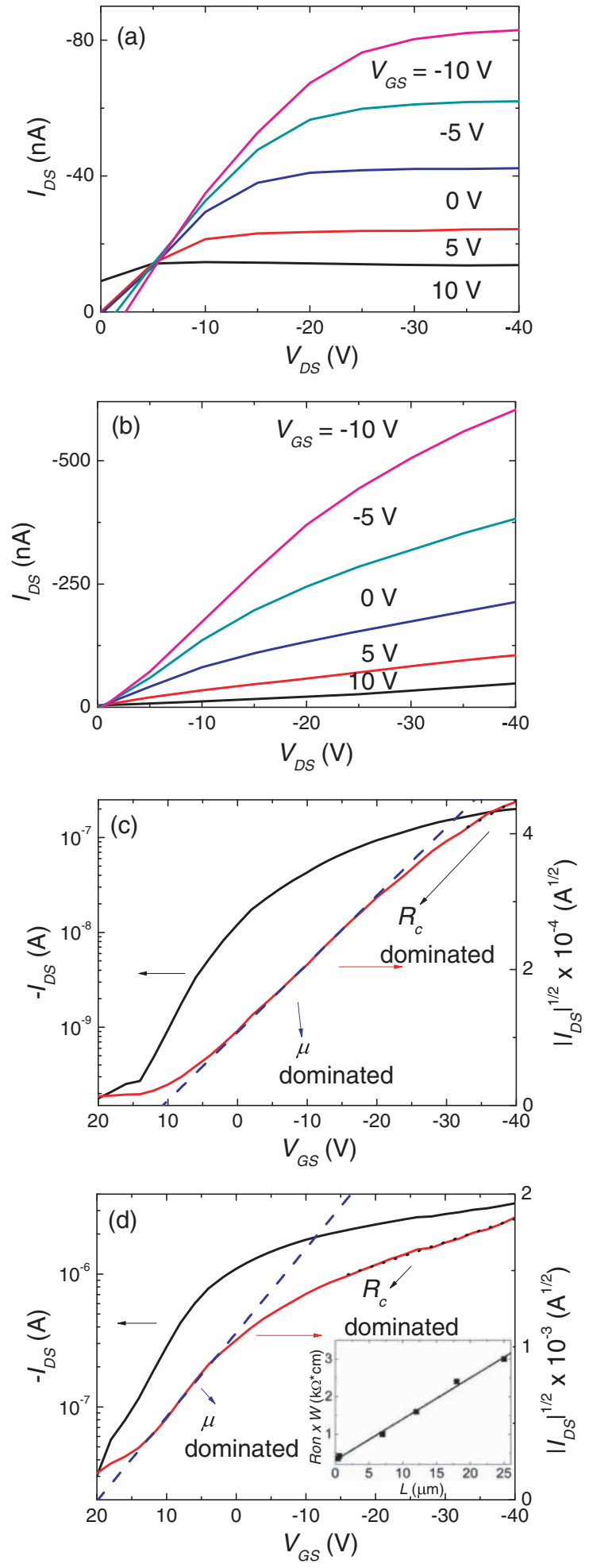

Figure 7. $a, b)$ : Output characteristics of microgaps and nanogaps. a) Microgap $(L=25 \mu \mathrm{m}, W=100 \mu \mathrm{m})$. b) SNL-made nanogap $(L=$ $400 \mathrm{~nm}, W=100 \mu \mathrm{m})$ P3HT FETs. c,d) Corresponding transfer characteristics, $I_{D S}$ (left vertical scale) and $\left|I_{D S}\right|^{1 / 2}$ (right scale) vs. $V_{G S}$, for $V_{D S}=-40 \mathrm{~V}$. Dashed and dotted lines indicate transistor- and contactdominated regimes, respectively. Inset of d): $R_{\mathrm{ON}} \cdot \mathrm{W}$ vs. $L$ (at $V_{\mathrm{GS}}=$ $-10 \mathrm{~V}$ and $V_{D S}=-5 \mathrm{~V}$ ). The superimposed line is best fit to experimental data (see the text). transfer characteristics, ${ }^{[25]}$ of about 110 , and a threshold voltage $\left(V_{\mathrm{TH}}\right)$ of $20 \mathrm{~V}$.

For microgap FETs, linear and saturation mobility $(\mu)$ of $10^{-3} \mathrm{~cm}^{2} \mathrm{~V}^{-1} \mathrm{~s}^{-1}$ and $10^{-2} \mathrm{~cm}^{2} \mathrm{~V}^{-1} \mathrm{~s}^{-1}$ are estimated by the device characteristics, ${ }^{[25]}$ whereas a kink is appreciable in the transfer curve of nanogap devices instead of a clear linear behavior. Standard FET characteristics, ${ }^{[25]} I_{\mathrm{DS}}^{(\text {linear })}=\frac{W}{L} C \mu\left(V_{\mathrm{GS}}-V_{\mathrm{TH}}\right) V_{\mathrm{DS}}$ and $I_{\mathrm{DS}}^{\text {(satur.) }}=\frac{W}{2 L} C \mu\left(V_{\mathrm{GS}}-V_{\mathrm{TH}}\right)^{2}$, where $C$ is the gate dielectric capacitance per unit area, are used to estimate the charge carrier mobility in microgap devices in the linear and saturation regime, respectively. In contrast, a model for the linear and saturation regime of short-channel transistors is found to extend the gradual channel approximation model by introducing ohmic contact resistances in series at source $\left(R_{\mathrm{S}}\right)$ and drain $\left(R_{\mathrm{D}}\right)$, as follows: ${ }^{[26]}$

$$
\begin{aligned}
& I_{\mathrm{DS}}^{(\text {linear) }} \\
& =-\frac{W}{L} C \mu\left[\left(V_{\mathrm{GS}}-R_{\mathrm{S}} I_{\mathrm{DS}}\right)-V_{\mathrm{TH}}-\frac{V_{\mathrm{DS}}-\left(R_{\mathrm{S}}+R_{\mathrm{D}}\right) I_{\mathrm{DS}}}{2}\right] \\
& \quad \times\left[V_{\mathrm{DS}}-\left(R_{\mathrm{S}}+R_{\mathrm{D}}\right) I_{\mathrm{DS}}\right] \\
& I_{\mathrm{DS}}^{\text {(satur.) }}=-\frac{W}{2 L} C \mu\left[\left(V_{\mathrm{GS}}-R_{\mathrm{S}} I_{\mathrm{DS}}\right)-V_{\mathrm{TH}}\right]^{2}
\end{aligned}
$$

Neglecting the term, $R_{\mathrm{S}} I_{\mathrm{DS}}$, the equation for the description of the linear regime can be rewritten as:

$$
\begin{aligned}
& I_{\mathrm{DS}}^{(\text {linear) }} \\
& \quad=-\frac{W}{L} C \mu\left[\frac{\left.\left[V_{\mathrm{GS}}-V_{\mathrm{TH}}-V_{\mathrm{DS}}\right) 2\right]}{\left.\left.1-r_{\mathrm{C}} C \mu\right) L \times\left[V_{\mathrm{GS}}-V_{\mathrm{TH}}-V_{\mathrm{DS}}\right) 2\right]}\right]
\end{aligned}
$$

where $r_{\mathrm{C}}=\mathrm{W} \times R_{\mathrm{C}}=W \times\left(R_{\mathrm{S}}+R_{\mathrm{D}}\right)$. The critical gate voltage, $\mathrm{V}_{\mathrm{GSX}}$, can be defined as the $V_{\mathrm{GS}}$ value where the channel resistance $\left(R_{\mathrm{CH}}\right)$ equals $R_{\mathrm{C}}: V_{\mathrm{GSX}}=V_{\mathrm{TH}}+\frac{V_{\mathrm{DS}}}{2}-\frac{L}{r_{\mathrm{C}} C \mu}$. From this expression, one finds $V_{\mathrm{GSX}} \cong 0 \mathrm{~V}$ for the nanogap devices realized here (Figure $7 \mathrm{~d}$ ). The mobility values are estimated by fitting experimental data by Equation 2 and 3, and found to be $10^{-3}$ and $10^{-4} \mathrm{~cm}^{2} \mathrm{~V}^{-1} \mathrm{~s}^{-1}$ in the saturation and in the linear regime, respectively. For gate voltages above $V_{\mathrm{GSX}}, R_{\mathrm{CH}}$ is sufficiently decreased by channel opening, and the device operation is dominated by the contact resistance (dotted line in Figure 7d).

We further investigate the contact properties of the conjugated polymer on electrodes, calculating $R_{\mathrm{C}}$ by channel length scaling analysis (transfer line method). ${ }^{[27]}$ The $L$-dependence of the device resistance in the linear regime can be approximated as: ${ }^{[28]} R_{\mathrm{ON}}=\left.\frac{\partial V_{\mathrm{DS}}}{\partial I_{\mathrm{DS}}}\right|_{V_{\mathrm{DS}}} ^{V_{\mathrm{GS}}}=R_{\mathrm{CH}}+R_{\mathrm{C}}=\frac{L}{\mathrm{~W} \mu C\left(V_{\mathrm{GS}}-V_{\mathrm{TH}}\right)}+R_{\mathrm{C}}$, where $R_{\mathrm{C}}$ depends on $L$. The inset of Figure $7 \mathrm{~d}$ shows a plot of $R_{\mathrm{ON}} \times$ $W$ vs. $L$ (at $V_{\mathrm{GS}}=-10 \mathrm{~V}$ and $V_{\mathrm{DS}}=-5 \mathrm{~V}$ ), which is well-fitted by the previous equation with an $R_{\mathrm{C}}$ value of $31 \mathrm{k} \Omega$. This value is comparable to that of bottom-contact P3HT transistors with $\mathrm{Cr} /$ $\mathrm{Au}$ electrodes made by photolithography. ${ }^{[29]}$

Though presenting mobility and $I_{\text {on }} / I_{\text {off }}$ ratio one order of magnitude lower than those of microgap devices, FETs with nanogaps are predicted to enable operation at $\mathrm{MHz}$ regimes, since the transition frequency, $f_{\mathrm{T}}=\frac{g_{m}}{2 \pi C W L} \cong \frac{\mu}{2 \pi L^{2}} V_{\mathrm{DS}}$, where $g_{m}$ is the transconductance, ${ }^{[24]}$ is expected to be around $1 \mathrm{kHZ}$ 

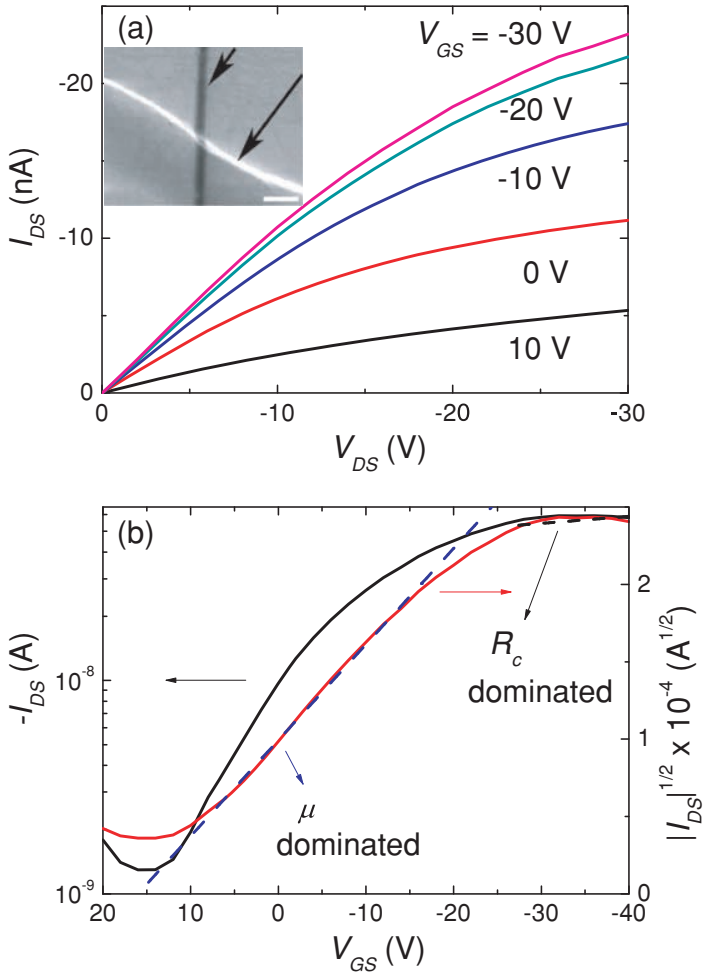

Figure 8. a) Output characteristics of a $\mathrm{P} 3 \mathrm{HT}$ nanofiber transistor $(L \cong$ $W \cong 250 \mathrm{~nm}$ ). Inset: device SEM image. Bar $=1 \mu \mathrm{m}$. The short and the long arrows indicate the gap and the fiber, respectively. b) Corresponding transfer characteristics, $I_{\mathrm{DS}}$ (left vertical scale) and $\left|I_{\mathrm{DS}}\right|^{1 / 2}$ (right scale), for $V_{D S}=-40 \mathrm{~V}$. Dashed and dotted lines are as in Figure 7.

and $1 \mathrm{MHz}$ for microgap and nanogap devices, respectively. The resulting increased switching speed is particularly promising for novel polymer radio frequency identification devices (RFIDs). ${ }^{[30]}$

In addition, the channel width can be substantially reduced using P3HT nanofibers as an active medium. Those can be realized by SNL on organics and PRINT as illustrated in Figure 1f - 1i. Analogously to FETs on nanogap electrodes (Figure 7b), nanofiber transistors with channel length and width of $250 \mathrm{~nm}$ (inset of Figure 8a) do not exhibit saturation up to $V_{\mathrm{DS}} \sim-30 \mathrm{~V}$ and for $V_{\mathrm{GS}}$ decreasing from +10 to $-30 \mathrm{~V}$ (Figure $8 \mathrm{a}$ ). Additionally, the transfer characteristics (Figure $8 \mathrm{~b}$ ) are similar to those of the nanogap FETs, with measured mobility and threshold voltage of $2.5 \times 10^{-3} \mathrm{~cm}^{2} / \mathrm{Vs}$ and $16 \mathrm{~V}$, respectively, and $I_{\mathrm{on}} / I_{\text {off }}$ $\sim 50$. This reduced value for the switching ratio is likely related to impurities contained in the polymer, or to contaminations such as oxygen and moisture introduced in the organic semiconductor ${ }^{[31]}$ due to exposure to air during PRINT. Finally, a $f_{\mathrm{T}}$ value as high as $15 \mathrm{MHz}$ is estimated for fiber devices by the usual expression of the transition frequency. ${ }^{[24]}$

\section{Conclusions}

In summary, SNL based on polymer fibers is a promising and cheap patterning route. We demonstrate differently shaped features on $\mathrm{Au}$ and elastomers with resolution down to $50 \mathrm{~nm}$. Polymer and nanofiber FETs are realized on SNL-made gap electrodes, with predictableelectrical characteristics. This versatile technology opens a new way for fabricating polymer nanoscale electronic devices.

\section{Experimental Section}

Materials: $n$-type Si(100) wafers with low resistivity $(<6 \mathrm{~m} \Omega \cdot \mathrm{cm})$ and coated by a thermally grown $\mathrm{SiO}_{2}$ layer with a thickness of $100 \mathrm{~nm}$ were provided by Silicon Materials. PMMA (120 kg mol-1, Sigma-Aldrich) was dissolved in formic acid (Fluka). PDMS (Sylgard 184) was purchased from Dow Corning. PFPE-urethane-methacrylate was kindly provided by Solvay Solexis, and the 2-hydroxy-2-methylpropriophenone photoinitiator (Darocur 1173) used for fabricating PFPE elements was from Ciba. P3HT $\left(87 \mathrm{~kg} \mathrm{~mol}^{-1}\right)$ was purchased from Sigma-Aldrich, and chloroform and toluene from J. T. Baker.

Electrospinning: A $0.1 \mathrm{~m} \mathrm{~m}$ formic acid solution of PMMA was placed into a $1.0 \mathrm{~mL}$ plastic syringe tipped with a 23-gauge stainless steel needle. The positive lead from a high voltage supply (XRM30P, Gamma High Voltage Research Inc.) was connected to the metal needle applying a bias in the range of 5-10 kV. The solution was injected at the end of the needle at a constant rate of $10 \mu \mathrm{L} \mathrm{min}{ }^{-1}$ by a syringe pump (33 Dual Syringe Pump, Harvard Apparatus Inc.), which prevents dripping at the end of the metallic capillary. The collector was placed at a distance of $10-15 \mathrm{~cm}$ from the needle and biased at a negative voltage of $-6 \mathrm{kV}$, while the collection time was varied from 30 to $60 \mathrm{~s}$ depending on the desired fiber density in the array. Aligned arrays of free-standing fibers are deposited by using $6 \mathrm{~m} \Omega$ two parallel Al-coated glass slides with a $1.5 \mathrm{~cm}$-wide gap as a collector, and then transferred on the cleaned $\mathrm{SiO}_{2}$ top surface of a $1 \mathrm{~cm} \times 1 \mathrm{~cm}$ slab of $\mathrm{n}$-type $\mathrm{Si}$ wafer.

SNL on Inorganic Surfaces: A thin metal layer $(5 \mathrm{~nm} / 45 \mathrm{~nm} \mathrm{Cr} / \mathrm{Au})$ was deposited on the sample surface by a PVD 75 equipment (Kurt J. Lesker) through shadow masks ("Micron"Cu grids with bars from 7 to $25 \mu \mathrm{m}$ in width and a matrix of $16 \times 16$ square holes with side of $100 \mu \mathrm{m}$, TAAB Laboratories Equipment Ltd). Thereafter, polymer fibers were detached by oxygen plasma (600 s at $150 \mathrm{~W}$, Tucano, Gambetti Kenologia) or by sonication in isopropanol for a few seconds, thus finally exposing $\mathrm{SiO}_{2}$ nanoscale windows.

SNL on Organic Surfaces: PMMA nanofiber arrays were treated by HDMS (Rohm and Haas Electronic Materials Ltd) vapor to form an antiadhesion layer for favoring the following mechanical peeling-off. Then, a thin layer of PDMS (A:B 1:9) is cast onto the array and polymerized in situ at $75{ }^{\circ} \mathrm{C}$ for $30 \mathrm{~min}$. As an alternative, a PFPE-prepolymer was obtained by mixing the PFPE-urethane dimethacrylate with $4 \% \mathrm{w} / \mathrm{w}$ of the photoinitiator, and PFPE molds were produced by spin-coating the prepolymer on the fibers array ( $400 \mathrm{rpm}$ for $40 \mathrm{~s}$ ) and irradiating it with UV light $(\lambda \sim 360 \mathrm{~nm}$, delivered by two $6 \mathrm{~W}$ lamps for $30 \mathrm{~s}$ at a distance of $10 \mathrm{~cm}$ from the sample), under an inert nitrogen atmosphere.

SEM and AFM: SEM was carried out on electrospun polymer fibers, SNL-made gap electrodes, and PDMS features by a Raith 150 electronbeam system operating with an acceleration voltage $(B)$ of $10 \mathrm{kV}$ and an aperture size of $30 \mu \mathrm{m}$. The fiber cross-section is investigated using $B=2 \mathrm{kV}$. PDMS surfaces were coated by $10 \mathrm{~nm}$ of $A u$ before investigation. AFM is performed by a Nanoscope IIla controller with Multimode head (Veeco). Phosphorous-doped Si tips were employed with an 8-10 nm nominal curvature radius and a resonant frequency of $250 \mathrm{kHz}$.

Transistor Fabrication: A thin layer of HMDS was spin-cast (4000 rpm, $40 \mathrm{~s}$ ) on both SNL-made nanogap, and on microgap electrodes defined by shadow masks ( $\mathrm{Cu}$ grids with $7-25 \mu \mathrm{m}$ bars and $100 \mu \mathrm{m}$ holes, TAAB Laboratories) to optimize the interface between the organic semiconductor and the gate dielectrics. Polymer FETs were realized by a $50 \mathrm{~nm}$ thick P3HT film, spin-coated $(2000 \mathrm{rpm}, 30 \mathrm{~s})$ from a $23 \mu \mathrm{m}$ chloroform solution. For nanofiber transistors, a $1 \mu \mathrm{L}$ droplet of P3HT solution ( $23 \mu \mathrm{M}$ in toluene) was deposited between a PFPE mold and a substrate with nanogap electrodes (Figure $1 \mathrm{~h}$ ). The P3HT solution, which is non-wetting for PFPE, remained confined within the recessed features of the mold. This was removed after about $12 \mathrm{~h}$, leaving P3HT 
nanofibers deposited across the nanogaps. All the transistors were annealed in an $\mathrm{N}_{2}$ atmosphere at $90{ }^{\circ} \mathrm{C}$ for $60 \mathrm{~min}$ to remove residual solvent and enhance material adhesion.

Electrical Characterization: A probe station (PH100, Süss Micro Tec $\mathrm{AG}$ ) and a stereomicroscope (MZ16FA, Leica Microsystems GmbH) were used for electrically contacting transistors. The characterization was carried out in air at room temperature and in dark, collecting and analyzing signals by means of a semiconductor parameter analyzer (4200 SCS, Keithley)

\section{Acknowledgements}

We gratefully acknowledge the financial support from the Regional Strategic Project "Ponamat" (PS_016) and the FIRB Contract RBIP06SH3W. This work is also performed in the framework of the FIRB RBFR08DJZI "Futuro in Ricerca". Dr. A. Zompatori and Solvay-Solexis are acknowledged for kindly providing the perfluoropolyether-urethane dimethacrylate elastomer.

Received: September 10, 2010

Revised: November 18, 2010 Published online: March 1, 2011

[1] M. Hamedi, K. Tvingstedt, R. H. Karlsson, P. Åsberg, O. Inganäs, Nano Lett. 2009, 9, 631.

[2] E. Mele, A. Camposeo, M. De Giorgi, F. Di Benedetto, C. De Marco, V. Tasco, R. Cingolani, D. Pisignano, Small 2008, 4, 1894.

[3] S. J. Maerkl, S. R. Quake, Science 2007, 315, 233.

[4] J. P. Renault, A. Bernard, D. Juncker, B. Michel, H. R. Bosshard, E. Delamarche, Angew. Chem. Int. Ed. 2002, 41, 2320.

[5] T. M. Squires, S. R. Quake, Rev. Mod. Phys. 2005, 77, 977.

[6] P. F. Murphy, K. J. Morton, Z. Fu, S. Y. Chou, Appl. Phys. Lett. 2007, 90, 203115.

[7] M. D. Austin, W. Zhang, H. Ge, D. Wasserman, S. A. Lyon, S. Y. Chou, Nanotechnology 2005, 16, 1058.

[8] Y. Xia, G. M. Whitesides, Angew. Chem. Int. Ed. 1998, 37, 550.

[9] J. P. Rolland, E. C. Hagberg, G. M. Denison, K. M. Carter, J. M. De Simone, Angew. Chem. Int. Ed. 2004, 43, 5796

[10] B. W. Maynor, I. LaRue, Z. Hu, J. P. Rolland, A. Pandya, Q. Fu, J. Liu, R. J. Spontak, S. S. Sheiko, R. J. Samulski, E. T. Samulski, J. M. DeSimone, Small 2007, 3, 845.
[11] F. Hua, Y. Sun, A. Gaur, M. A. Meitl, L. Bihaut, L. Rotkina, J. Wang, P. Geil, M. Shim, J. A. Rogers, Nano Lett. 2004, 4, 2467.

[12] E. P. De Poortere, H. L. Stormer, L. M. Huang, S. J. Wind, S. O'Brien, M. Huang, J. Hone, J. Vac. Sci. Technol. B 2006, 24, 3213.

[13] D. H. Reneker, I. Chun, Nanotechnology 1996, 7, 216.

[14] D. A. Czaplewski, J. Kameoka, R. Mathers, G. W. Coates, H. G. Craighead, Appl. Phys. Lett. 2003, 83, 4836.

[15] S. Pagliara, A. Camposeo, A. Polini, R. Cingolani, D. Pisignano, Lab Chip 2009, 9, 2851.

[16] Y. Ishii, H. Sakai, H. Murata, Mater. Lett. 2008, 62, 3370.

[17] N. Y. Lee, Y. S. Kim, Nanotechnology 2007, 18, 415303.

[18] D. Pisignano, L. Persano, G. Gigli, R. Cingolani, F. Babudri, G. M. Farinola, F. Naso, Appl. Phys. Lett. 2004, 84, 1365.

[19] H. Dong, V. Nyame, A. G. MacDiarmid, W. E. Jones Jr., J. Polym. Sci., Part B: Polym. Phys. 2004, 42, 3934.

[20] S. Pagliara, A. Camposeo, R. Cingolani, D. Pisignano, Appl. Phys Lett. 2009, 95, 263301.

[21] A. Camposeo, F. Di Benedetto, R. Stabile, R. Cingolani, D. Pisignano, Appl. Phys. Lett. 2007, 90, 143115.

[22] A. Camposeo, F. Di Benedetto, R. Stabile, A. A. R. Neves, R. Cingolani, D. Pisignano, Small 2009, 5, 562.

[23] F. Di Benedetto, A. Camposeo, S. Pagliara, E. Mele, L. Persano, R. Stabile, R. Cingolani, D. Pisignano, Nat. Nanotechnol. 2008, 3, 614

[24] S. M. Sze, K. K. Ng, in Physics of Semiconductor Devices, 3rd Edition(Eds. S. M. Sze, K. K. Ng), Wiley-Interscience, Hoboken, NJ 2006, Ch. 6.

[25] IEEE Std 1620-2008, IEEE Standard for Test Methods for the Characterization of Organic Transistors and Materials, http://standards. ieee.org/.

[26] A. Hoppe, T. Balster, T. Muck, V. Wagner, Phys. Status Solidi A 2008 205, 612.

[27] S. Luan, G. W. Neudeck, J. Appl. Phys. 1992, 72, 766.

[28] Q. J. Cai, M. B. Chan-Park, Q. Zhou, Z. S. Lu, C. M. Li, B. S. Ong, Org. Electron. 2008, 9, 936.

[29] L. Bürgi, T. J. Richards, R. H. Friend, H. Sirringhaus, J. Appl. Phys 2003, 94, 6129.

[30] S. Steudel, K. Myny, V. Arkhipov, C. Deibel, S. De Vusser, J. Genoe P. Heremans, Nat. Mater. 2005, 4, 597.

[31] H. Liu, C. H. Reccius, H. G. Craighead, Appl. Phys. Lett. 2005, 87, 253106 